\title{
Island nucleation in thin-film epitaxy: A first-principles investigation
}

\author{
Kristen A. Fichthorn ${ }^{1}$ and Matthias Scheffler ${ }^{2}$ \\ ${ }^{1}$ Department of Chemical Engineering, The Pennsylvania State University, University Park, PA 16802, USA \\ ${ }^{2}$ Fritz-Haber-Institut der Max-Planck-Gesellschaft, Faradayweg 4-6, D-14195 Berlin, Germany
}

\begin{abstract}
We describe a theoretical study of the role of adsorbate interactions in island nucleation and growth, using $\mathrm{Ag} / \mathrm{Pt}(111)$ heteroepitaxy as an example. From density-functional theory, we obtain the substrate-mediated $\mathrm{Ag}$ adatom pair interaction and we find that, past the short range, a repulsive ring is formed about the adatoms. The magnitude of the repulsion is comparable to the diffusion barrier. In kinetic Monte Carlo simulations, we find that the repulsive interactions lead to island densities over an order of magnitude larger than those predicted by nucleation theory and thus identify a severe limitation of its applicability. Copyright 2000 by The American Physical Society.
\end{abstract}

PACS numbers: 68.55.-a, 61.43.Hv, 68.35.Fx, 82.20.Mj

Island nucleation is often the first step in thin-film epitaxy and is, thus, relevant to the synthesis of a wide variety of interfacial materials. Achieving a quantitative understanding of the island morphologies (i.e., sizes, shapes, density, spatial distribution, etc.) that develop in the initial stages of thin-film growth is also important for fundamental reasons. Since thin-film epitaxy frequently occurs away from equilibrium, the kinetics of deposition and surface diffusion play a key role in governing island morphology and there is great variety in the resulting structures. Considering shapes [1] 8], for example, islands can be fractal-like or compact and triangular, hexagonal, square, rectangular, etc.. Each of these structures is a signature of an intricate kinetic balance and reflects a complex set of interatomic interactions that is unique for each material.

Despite the complexity and potentially enormous variety in growth morphologies, certain aspects of island nucleation and growth appear to be common to many different systems. In a general description, gas-phase species are deposited onto an initially bare solid substrate with a rate $F$. These species hop on the surface with a rate $D=\nu_{0} e^{-E_{b}^{0} / k_{B} T}$, where $\nu_{0}$ is the preexponential factor, $E_{b}^{0}$ is the diffusion-energy barrier for an isolated species, $k_{B}$ is Boltzmann's constant, and $T$ is temperature. Hopping mediates the aggregation of adspecies into nuclei, which either dissociate with an energy barrier $E_{d, i}$, if they are below a critical size $i$, or grow subsequently to become stable islands. Initially, the formation of island nuclei is the main process taking place. As the surface coverage increases, it becomes increasingly likely that deposited species will add to stable islands and promote their growth instead of forming new nuclei. These general features can be captured in a mean-field theory for the stable island density $N_{x}$ [9]. In the island growth regime, this expression has the form

$$
N_{x} \sim(F / D)^{i /(i+2)} \exp \left(-E_{d, i} / k_{B} T\right)^{1 /(i+2)} .
$$

Although the utility of a general expression cannot be overstated, Eq. 1 1 cannot describe all aspects of thin-film epitaxy and it is important to understand its limitations. In the interest of achieving a complete and predictive model for thin-film morphology, it is clearly desirable to have an approach that is as free as possible from arbi- trary parameters or assumptions. In this Letter, with an aim toward this ideal approach, we present the results of a combined kinetic Monte Carlo (kMC) and firstprinciples, density-functional theory (DFT) study of island nucleation in a model for the growth of $\mathrm{Ag}$ on a monolayer (ML) of $\mathrm{Ag}$ on $\mathrm{Pt}(111)$. Our choice of this model system was motivated by intriguing results from recent, low-temperature, scanning-tunneling microscopy (STM) studies [10 12], in which Eq. 11 was used to obtain the energy barrier and preexponential factor for adatom hopping. Shown in Table I are the parameters obtained in these studies.

A striking feature of the experimental results is that the preexponential factors are significantly smaller than would be anticipated for systems such as these. For example, from $a b$ initio calculations, Ratsch and Scheffler 113] find a preexponential factor of $\nu_{0}=1.3 \times 10^{12} \mathrm{~s}^{-1}$ for a $\mathrm{Ag}$ adatom on 1-ML- $\mathrm{Ag} / \mathrm{Pt}(111)$, with a diffusion barrier of $E_{b}^{0}=63 \mathrm{meV}$. Inserting the experimental and theoretical values for the diffusion parameters into Eq. 1 in the low-temperature limit where $i=1$ and $E_{d, i}=0$, we see that the experimental island densities are about an order of magnitude higher than predictions based on the theoretical diffusion parameters. Here, we investigate the origins of this discrepancy. Our DFT-kMC model includes many features of the complex potential-energy surface experienced by $\mathrm{Ag}$ adatoms during thin-film growth and is free from several of the assumptions in Eq. 1. We find that one of these assumptions - that interactions between adsorbed species do not extend beyond a short range - is violated. For systems with low diffusionenergy barriers [such as $\mathrm{Ag}$ on 1-ML- $\mathrm{Ag} / \mathrm{Pt}(111)]$, we show that these long-range, adatom-adatom interactions play an important and previously underestimated role in island nucleation and growth.

\begin{tabular}{c|c|c|r}
\hline \hline System & $E_{b}^{0}(\mathrm{meV})$ & $\nu_{0}\left(\mathrm{~s}^{-1}\right)$ & Ref. \\
\hline $\mathrm{Al}$ on $\mathrm{Au}(111)$ & 30 & $7 \times 10^{3}$ & 10 \\
\hline $\mathrm{Al}$ on $\mathrm{Al}(111)$ & 42 & $8 \times 10^{6}$ & \\
\hline $\mathrm{Ag}$ on $1-\mathrm{ML}-\mathrm{Ag} / \mathrm{Pt}(111)$ & 60 & $10^{9}$ & 12 \\
\hline \hline
\end{tabular}

TABLE I. Experimentally determined diffusion-energy barriers and preexponential factors. 
The DFT calculations 14 are performed using the plane-wave, pseudopotential |15 method within the generalized gradient approximation [16]. Previously, Ratsch et al. [17] showed in DFT calculations that the diffusionenergy barrier of an $\mathrm{Ag}$ atom on the 1-ML-Ag/Pt(111) substrate is essentially the same as that on a strained $\mathrm{Ag}(111)$ substrate, in which $\mathrm{Ag}$ is given the lattice constant of Pt. Thus, to model the heteroepitaxial system we use strained $\operatorname{Ag}(111)$, in which the lattice constant is set to a value of $4.01 \AA$. This value is 4.61 percent smaller than our calculated lattice constant for bulk Ag. We use the supercell approach to describe the surface, which is modeled as a $(4 \times 4 \times 4)$ slab with a vacuum spacing of five interlayer distances. The cut-off energy is $50 \mathrm{Ry}$ and we use $4 \mathbf{k}$ points to sample the full surface Brillouin zone. The top layer of a bare slab is fully relaxed. Subsequently, an adatom is placed in a binding site (fcc and hcp three-fold hollow sites), and its height is optimized with respect to the fixed substrate. To calculate adatom interaction energies, two (or more) adatoms are placed on the relaxed (and fixed) substrate with heights fixed to values from the single-adatom calculations. In this way, we seek to isolate the electronic interaction between adatoms in binding sites. With simultaneous relaxation of both the adatoms and surface atoms, we can resolve the role of substrate-mediated, elastic interactions in the total interaction energy. Full relaxation of a few trial structures and inspection of the forces in our partially relaxed slabs indicates that elastic interactions are not highly dependent on adsorbate configuration and that our results will change by $10 \mathrm{meV}$ or less with full relaxation.

The total interaction energy $\Delta E$ for a periodic slab containing $N$ adatoms, of which $M$ are at binding site $a$ and $(N-M)$ are at binding site $b$, is given by $\Delta E=$ $E_{S+N}^{a, b}-M E_{S+1}^{a}-(N-M) E_{S+1}^{b}+(N-1) E_{S}$. Here, $E_{S+N}^{a, b}$ is the total energy of a slab with $N$ adatoms, $E_{S+1}^{a}$ and $E_{S+1}^{b}$ are the total energies of slabs containing one adatom, and $E_{S}$ is the total energy of a bare slab. In the DFT supercell approach, the total interaction energy is comprised of interactions between different adatoms in the slab and interactions between adatoms in the slab and the periodic-image adatoms. We can express $\Delta E$ as a function of these interactions using the lattice-gas Hamiltonian approach (see, e.g. [18]), which yields

$$
\begin{aligned}
\Delta E= & \frac{1}{2} \sum_{i, j} V^{(2)}\left(\mathbf{R}_{i, j}\right) n_{i} n_{j} \\
& +\frac{1}{3} \sum_{i, j, k} V^{(3)}\left(\mathbf{R}_{i, j}, \mathbf{R}_{i, k}\right) n_{i} n_{j} n_{k}+\ldots
\end{aligned}
$$

Here, the summations run over all sites $i$ in the slab and all sites $j$ and $k$ in the supercell (which includes both the slab and its periodic images), $n_{m}$ is unity if site $m$ $(m=i, j, k)$ is occupied and zero, otherwise, $V^{(2)}\left(\mathbf{R}_{i, j}\right)$ is the pair interaction between two adatoms on sites $i$ and $j$, and $V^{(3)}\left(\mathbf{R}_{i, j}, \mathbf{R}_{i, k}\right)$ is the trio interaction between three adatoms on sites $i, j$, and $k$. We neglect higher-order interactions. Another assumption implicit in Eq. 2 is that the interaction between adatoms at a fixed distance is independent of whether these atoms occupy fcc or hcp sites. We confirmed this assumption in one trial calculation. Finally, the adatom binding energies on fcc and hcp sites are virtually equal: The fcc site is favored by less than $3 \mathrm{meV}$.

Thus, for a given adatom configuration, we express $\Delta E$ as a sum of pair and trio interactions with unknown coefficients. From 18 different configurations, we obtain a system of linear equations and solve these for pairinteraction coefficients up to the $13^{\text {th }}$-neighbor, as well as for 5 different trio interactions. We assume that all other interaction coefficients are zero. To verify our parameterization of Eq. (2), we used our interaction parameters to predict the total interaction energy in several, additional test structures. All of the predicted values agreed well with values from DFT calculations.

The pair interaction is shown in Fig. 1, where we also show results for $\mathrm{Ag}$ on unstrained $\mathrm{Ag}(111)$. For both surfaces, this interaction is strongly attractive at the nearest-neighbor distance and repulsive at longer distances. It is interesting to consider the origins of the long-range repulsion. At these distances, the interaction could be due to substrate-mediated elastic interactions or of electronic origin [19]. Since we find that elastic interactions play a small role here, the repulsion is primarily an electronic effect. Each adatom induces a small perturbation in the electron density, which decays with distance from the adatom in an oscillatory manner. The asymptotic tail, which is expected to decay with distance $d$ as $d^{-5}$ (or as $d^{-2}$, if a partially filled surface state is involved), is a Friedel-type oscillation. Friedel oscillations have been imaged as concentric, ring-like, features around defects in low-temperature STM studies of several noble-metal surfaces [including $\mathrm{Ag}(111)$ ] [20]. We expect the Friedel tail to extend to much longer distances than can be probed in DFT calculations. However, interactions associated with the Friedel tail should be weaker than those probed here. Thus, the central-ring interaction resolved here will have the most significant ramifications for thin-film morphology.

From Fig. 11(a), we see that the magnitude of the repulsive ring for $\mathrm{Ag}$ on strained $\mathrm{Ag}(111)$ is comparable to the diffusion-energy barrier for an isolated adatom. For $\mathrm{Ag}$ on $\mathrm{Ag}$ (111), it appears that the repulsive interaction is weaker and the diffusion-energy barrier is larger. The diffusion barriers reported here are obtained with full relaxation of both the first-layer slab atoms and the adatom. Our barriers are in good agreement with experimental values 12 for $\mathrm{Ag}$ on 1-ML-Ag/Pt(111) (60 meV) and on $\mathrm{Ag}(111)$ (97 meV) and with those of Ratsch and Scheffler 13]. If the interaction energy and the diffusion barrier are of comparable size, we expect interatomic interactions to significantly influence adatom diffusion and island formation. Since Eq. 11 neglects the influence of long-range interactions, it is unclear if this expression is accurate under these circumstances.

To resolve the effect of long-range interactions on thin- 


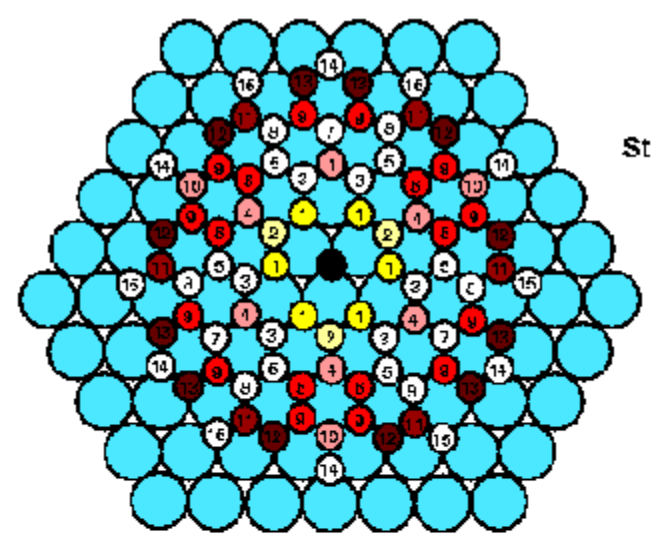

Energy : $m e V$

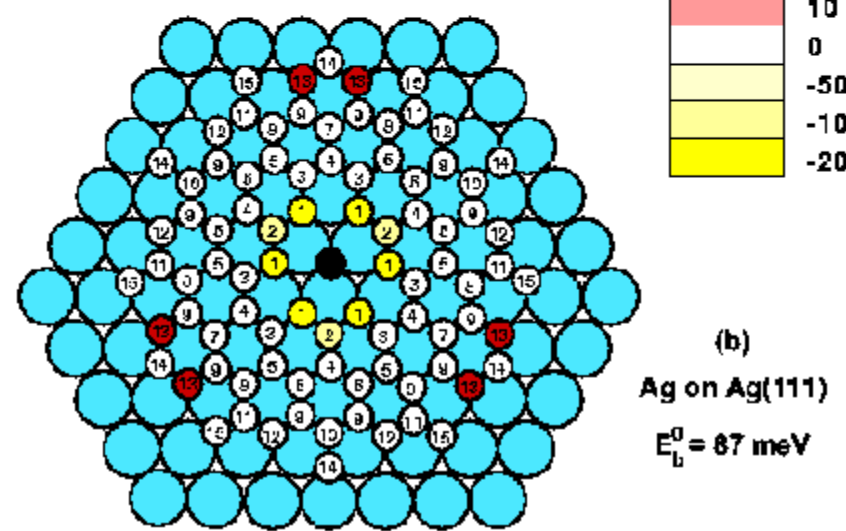

FIG. 1. Pair interaction energy as a function of distance from a central adatom, shown in black, for (a) $\mathrm{Ag}$ on strained $\mathrm{Ag}(111)$ and (b) $\mathrm{Ag}$ on $\mathrm{Ag}(111)$. Also noted are the diffusion-energy barriers, $E_{b}^{0}$, of isolated adatoms on these substrates.

film growth, we developed a kMC model employing the general method of Fichthorn and Weinberg [22] and incorporating the pair potential for $\mathrm{Ag}$ on strained $\mathrm{Ag}(111)$ shown in Fig. 1](a). In the initial stages of thin-film epitaxy, the surface coverage is low and pair interactions are likely to be the only significant interactions governing island nucleation and growth 21]. In our kMC model, atoms are deposited onto a fcc(111) substrate with a rate of $F=0.1 \mathrm{ML} / \mathrm{s}$. An adatom hops from site $i$ to site $j$ with a rate given by $D_{i \rightarrow j}=\nu_{0} e^{-E_{i \rightarrow j} / k_{B} T}$, where $E_{i \rightarrow j}$ is the energy barrier to hop from site $i$ to $j$. For the hopping-rate parameters, we use $\nu_{0}=10^{12} \mathrm{~s}^{-1}$ 13]. The energy barrier is given by $E_{i \rightarrow j}=E_{i, j}^{\ddagger}-E_{i}$, where $E_{i}$ is the energy with an atom at site $i$ and $E_{i, j}^{\ddagger}$ is the energy of the transition state between sites $i$ and $j$. In general, $E_{i, j}^{\ddagger}$ should depend on both $E_{i}$ and $E_{j}$. Considering possible permutations of adatom configurations with $13^{\text {th }}$ neighbor interactions, $\sim 10^{14}$ different, diffusion-energy barriers could occur. To make the problem tractable, we adopt a simple model, in which $E_{i \rightarrow j}=E_{b}^{0}+\frac{1}{2}\left(E_{j}-E_{i}\right)$. All of the quantities in this equation are obtained from DFT calculations. We have tested this equation for a trial geometry in which an adatom with four fcc $9^{\text {th }}$ neighbors hops to a nearest-neighbor hcp site where it has two $7^{\text {th }}$ and two $12^{\text {th }}$ neighbors. From our simple model, we find

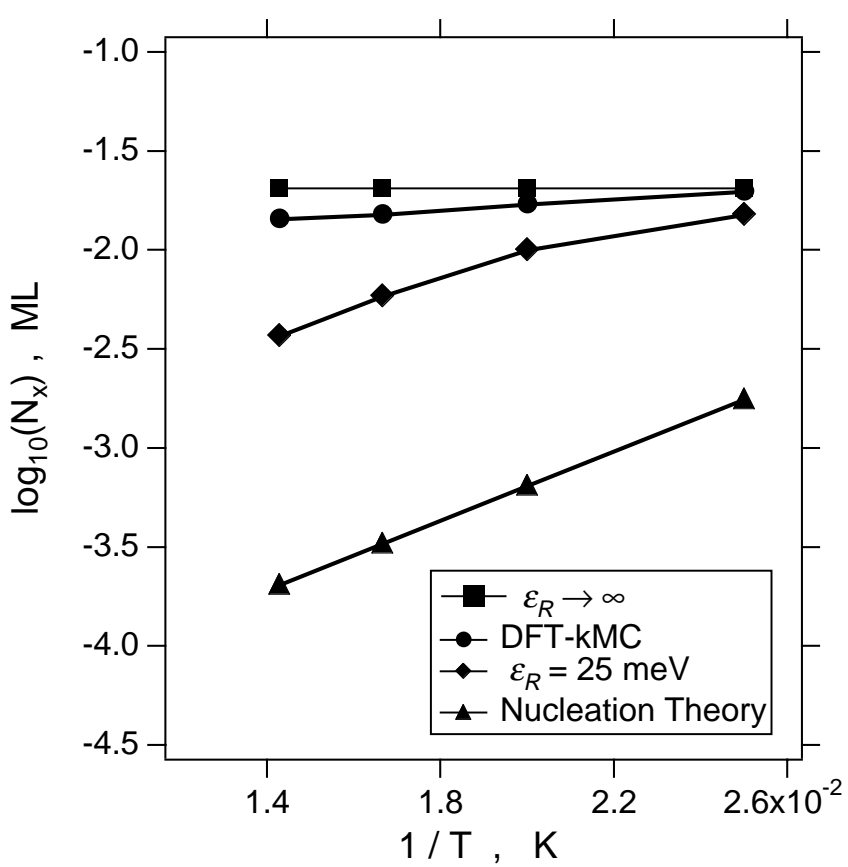

FIG. 2. Arrhenius plot of the island density as a function of temperature from an impermeable repulsive ring (squares), the DFT-kMC model (circles), repulsive ring with $\varepsilon_{R},=25$ meV (diamonds), and nucleation theory (triangles).

$E_{i \rightarrow j}=53 \mathrm{meV}$, which is in remarkable agreement with the value of $46 \mathrm{meV}$ from DFT calculations.

We simulated thin-film epitaxy over temperatures ranging from 40-70 $\mathrm{K}$ and determined island densities in the beginning of the island growth regime. These low temperatures are in the range of the experimental studies (cf., Table I). At such low temperatures, Eq. 1 reduces to the form $N_{x} \sim(F / D)^{1 / 3}$. Fig. 2 2 shows an Arrhenius plot of the island density from our DFT-kMC model as a function of temperature. Also shown in Fig. 2 is the island density predicted by nucleation theory for the values of $F, \nu_{0}$, and $E_{b}^{0}$ used here. To quantitatively compare nucleation theory with the simulations, a proportionality coefficient $\eta$ is needed in Eq. 1 (i.e., $N_{x}=\eta(F / D)^{1 / 3}$ ). This coefficient is related to the efficiency of the islands in capturing adatoms. Using a self-consistent approach, $\eta=0.25$ [23 and values of $\eta$ ranging from 0.2 to 0.23 have been found in $\mathrm{kMC}$ simulations of $\mathrm{Ag}$ island nucleation on $\operatorname{Pt}(111)$ 24]. Here, we use $\eta=0.25$.

In Fig. 2, we see that the DFT-kMC island densities are an order of magnitude (or more) above the theoretical values. To understand this, we construct a caricature model, in which we replace the set of pair interactions shown in Fig. 11(a) with a nearest-neighbor attractive interaction and a uniform, repulsive ring of strength $\varepsilon_{R}$ at distances 10-13. By varying the magnitude of $\varepsilon_{R}$, we span the entire range of possible behaviors in this system. As $\varepsilon_{R} \rightarrow \infty$, the island density assumes a constant, maximum value that is independent of temperature (cf., Fig. 2). This is because island nucleation can only occur when one atom is deposited within the repulsive ring of another and it is governed by the temperature-independent deposition rate. In this regime, many adatoms are isolated by 
repulsion in the initial stages of deposition. Each isolated adatom becomes a stable island when another atom is deposited into its ring and the resulting island density is significantly higher than in the absence of such a ring.

As $\varepsilon_{R}$ is decreased, diffusing adatoms are increasingly able to surmount the ring barrier and a second channel for island nucleation and growth (via long-range, adatom diffusion) opens up. The extent to which long-range diffusion contributes to island nucleation and growth depends on the temperature. In Fig. 2, we see that at $40 \mathrm{~K}$, the DFT-kMC island density is the same as that for an infinitely repulsive ring (i.e., diffusing adatoms are unable to penetrate the ring on the time scale for nucleation). As the temperature increases, adatoms are increasingly able to penetrate the ring barrier to aggregate and add to existing islands via long-range diffusion. Consequently, the island density decreases with increasing temperature. It is interesting to note that for the conditions studied, even a relatively weak repulsive ring with $\varepsilon_{R}=25 \mathrm{meV}$ can lead to significantly higher island densities than those predicted by nucleation theory.

Returning to our discussion of the experimental results shown in Table I, we point out that the order-ofmagnitude difference between the island densities predicted from $a b$ initio calculations [13] and those found experimentally for $\mathrm{Ag}$ on 1-ML- $\mathrm{Ag} / \mathrm{Pt}(111)$ 12] is also seen in our study, comparing the island densities predicted by nucleation theory to those found in our DFT-kMC "computer experiments" (cf., Fig. 2). Thus, we conclude that our results can explain the theoretical-experimental gap in the island density for $\mathrm{Ag}$ on 1-ML-Ag/Pt(111). Further, our results indicate that for $\mathrm{Ag}$ on $\mathrm{Ag}(111)$, the theoretical-experimental gap should be weaker or nonexistent. This result is also consistent with a comparison of theoretical diffusion parameters for $\mathrm{Ag}$ on $\mathrm{Ag}(111)$ [13, 17 $\left(\nu_{0}=8.2 \times 10^{11} \mathrm{~s}^{-1}, E_{b}^{0}=82 \mathrm{meV}\right)$ to experimental values [12 obtained using Eq. 1] $\left(\nu_{0}=2 \times 10^{11} \mathrm{~s}^{-1}\right.$, $\left.E_{b}^{0}=97 \mathrm{meV}\right)$. Finally, Bogicevic and co-workers 25 recently found similar DFT and kMC results for both $\mathrm{Al}(111)$ and $\mathrm{Cu}(111)$ homoepitaxy.

Thus, we conclude that long-range, electronic, substrate-mediated adatom interactions exist and, if their strength is comparable to the diffusion barrier, they can significantly influence surface diffusion and the growth morphology in thin-film epitaxy. For Ag on strained $\mathrm{Ag}(111)$, the adatom pair interaction becomes repulsive past the short range and the repulsion forms a ring around isolated adatoms. The magnitude of the repulsion is comparable to the diffusion barrier. By inhibiting island nucleation and growth via long-range adatom diffusion, these interactions lead to island densities that are substantially larger than those predicted by nucleation theory.

We acknowledge helpful conversations with A. Bogicevic, H. Brune, P. Kratzer, C. Ratsch, A. Seitsonen, and C. Stampfl. Support for this work is from the Alexander von Humboldt Foundation and the NSF (DMR-9617122).
[1] T. Michely, M. Hohage, M. Bott, and G. Comsa, Phys. Rev. Lett. 70, 3943 (1993).

[2] H. Brune, C. Romainczyk, and H. Röder, Nature 369, 469 (1994); H. Brune, H. Röder, and J. Nørskov, Surf. Sci. 349, L115 (1996).

[3] P. Ruggerone, C. Ratsch, and M. Scheffler, in Growth and Properties of Ultrathin Epitaxial Layers, Eds. D.A. King, D.P. Woodruff. The Chemical Physics of Solid Surfaces, Vol. 8. Elsevier Science, Amsterdam (1997), p 490.

[4] A. Bogicevic, J. Strömquist, and B. I. Lundqvist, Phys. Rev. Lett. 81, 637 (1998).

[5] S. Ovesson, A. Bogicevic, and B.I. Lundqvist, Phys. Rev. Lett. 83, 2608 (1999).

[6] J. A. Stroscio, D. T. Pierce, and R. A. Dragoset, Phys. Rev. Lett. 70, 3615 (1993).

[7] C.-M. Zhang, M. C. Bartelt, J.-M. Wen, J. W. Evans, and P. A. Thiel, J. Crystal Growth 174, 851 (1997).

[8] S. Günther, E. Kopatzki, M. C. Bartelt, J. W. Evans, and R. J. Behm, Phys. Rev. Lett. 73, 553 (1994).

[9] J. A. Venables, Phil. Mag. 27, 697 (1973).

[10] B. Fischer, H. Brune, J. V. Barth, A. Fricke, and K. Kern, Phys. Rev. Lett. 82, 1732 (1999).

[11] J. V. Barth, H. Brune, B. Fischer, J. Weckesser, and K. Kern, Phys. Rev. Lett. 84, 1732 (2000).

[12] H. Brune, et al., Phys. Rev. B 52, R14380 (1995).

[13] C. Ratsch and M. Scheffler, Phys. Rev. B 58, 13163 (1998).

[14] M. Bockstedte, A. Kley, J. Neugebauer, and M. Scheffler, Comput. Phys. Comm. 107, 187 (1997).

[15] M. Fuchs and M. Scheffler, Comput. Phys. Commun. 119, 67 (1999).

[16] J. P. Perdew, K. Burke and M. Ernzerhof, Phys. Rev. Lett. 77, 3865 (1996).

[17] C. Ratsch, A. P. Seitsonen, and M. Scheffler, Phys. Rev. B 55, 6750 (1997).

[18] C. Stampfl, H. J. Kreuzer, S. H. Payne, H. Pfnür, and M. Scheffler, Phys. Rev. Lett. 83, 2993 (1999).

[19] T. L. Einstein and J. R. Schrieffer, Phys. Rev. B 7, 3629 (1973); T. L. Einstein, Handbook of Surface Science, W. N. Unertl, Ed. (Elsevier, Amsterdam, 1996), Vol. 1, p. 577.

[20] See, e.g., B.G. Briner et al., Europhysics News 28, 97 (1997) and references therein.

[21] We find that trio interactions are attractive and significant at high adatom densities. They are unimportant at the medium and longer distances probed here and we will discuss them elsewhere.

[22] K. A. Fichthorn and W. H. Weinberg, J. Chem. Phys. 95, 1090 (1991).

[23] G. S. Bales and D.C. Chrzan, Phys. Rev. B 50, 6057 (1994).

[24] H. Brune, G. S. Bales, J. Jacobsen, C. Boragno, and K. Kern, Phys. Rev. B 60, 5991 (1999).

[25] A. Bogicevic, S. Ovesson, P. Hyldgaard, B. I. Lundqvist, and D. R. Jennison (preprint). 